\title{
EX FACTO IUS ORITUR*: PROCESOS DE ESCÁNDALO Y EL DERECHO MUNDIAL EMERGENTE
}

\author{
Andreas Fischer-Lescano*** \\ Universidad Jobann Wolfgang Goethe
}

RESUMEN. Con referencia al proceso transitorio de la Argentina, el artículo explora la génesis de un sistema transnacional del Derecho. Ese sistema no es formado exclusivamente por el Derecho internacional publico. Especialmente en el marco de los derechos humanos la sociedad civil globalizada es la fuerza central, participando en la creación de normas transnacionales. Las Madres de la Plaza de Mayo han iniciado un proceso de escandalización global, que ha resultado en la instauración de un nuevo régimen determinando que la desaparición es una violación de los derechos humanos universales.

Palabras clave: sistema transnacional de Derecho, derechos humanos, globalización.

ABSTRACT. Under reference to the transitional process in Argentina the article explores the emergence of a transnational system of law. This legal system does not only consist of «international public law». Especially in the field of human rights it is the global civil society which is the driving force behind processes of transnational legal norms creation. The Argentinean Madres de la Plaza de Mayo initialized a global scandalization process which resulted in the instauration of a new regime under which disappearance is a violation of universal human rights.

Keywords: transnational system of law, human rights, globalization.

\footnotetext{
* Ulpianus, Digesta, 44, 7, 25.

** Agradezco a Cristina Hoss, Michael KLODE, Tania LESCANO y a Paola Andrea LOMBARDI su colaboración.
} 


\section{EX FACTO}

L

a última dictadura argentina es considerada como una de las más crueles de América Latina. Se habla de aproximadamente 30.000 desaparecidos (CONADEP, 1984; Amnesty International, 1987). El denominado «Proceso de Reorganización Nacional» no es el único régimen que usó esa técnica, pero la Junta Militar argentina constituye un ejemplo único dada su crueldad, brutalidad y nefastas consecuencias.

La primera reacción en la opinión pública argentina fue el silencio; un «silencio social» (SONDERÉGUER, 1985: 8) que intentaron utilizar las autoridades militares encargadas de la «reestructuración». Pero el movimiento de derechos humanos se hizo paso a través de ese silencio y se erigió como abogado de una sociedad que no tenía otros caminos institucionales para brindar su oposición (SCHOLZ, 1990: 131).

\subsection{Lo Público en Argentina}

La protesta comenzó en la Plaza de Mayo, una plaza también utilizada en su momento por los propagandistas militares. Allí se juntaron las madres de los desaparecidos, con sus pañuelos blancos, para reclamar silenciosamente los crímenes cometidos contra sus hijos e hijas desaparecidos (HAUCK/HuHLE, 1996: 119). «[N]osotras golpeábamos, todas, las mismas puertas», así describió Hebe de Bonafini el comienzo poco espectacular de la protesta el 30 de abril de 1977 (BRUSCHTEIN, 1997). En sus propias palabras: «Todos ustedes saben que ahí nos conocimos; algunas en el Ministerio del Interior, algunas en la Policía, algunas en la calle, algunas en la desesperación de ir a la cárcel a ver si estaban ahí. Y a la Iglesia. Y un día, estando en la iglesia, en la iglesia de los asesinos, en la iglesia Stella Maris, que es la iglesia de la Marina, donde íbamos a ver a Gracelli, Azucena (Villaflor de Vincenti) dijo que ya basta, que no se podía más estar ahí, que ya no conseguíamos nada, que por qué no íbamos a la Plaza y hacíamos una carta para pedir audiencia, y que nos dijeran qué había pasado con nuestros hijos. Y así fuimos por primera vez un sábado. Nos dimos cuenta que no nos veía nadie, que no tenía ningún sentido. Era un 30 de abril. Decidimos volver a la otra semana un viernes. Y a la otra semana decidimos ir el jueves» (BONAFINI, 1988).

«Lo Público», que a los militares argentinos había preocupado tanto y que (a diferencia de Pinochet, que juntó 7.000 víctimas en el Estadio Nacional de Santiago) querían evitar absolutamente (MATTAROLLO, 1985: 10), comenzó a formarse y a nombrar ilegal lo que los militares denominaron «restauración del orden estatal» y «lucha legítima contra la subversión» ${ }^{1}$.

${ }^{1}$ Así, el Obispo Bonamín afirmó el 5 de diciembre de 1977 en una conferencia de la Universidad Nacional del Litoral, Paraná, Provincia de Entre Ríos: «El mundo está dividido por dos filosofías incompatibles, perfiladas por dos fronteras ideológicas: el materialismo ateo y el humanismo cristiano. Las Fuerzas Armadas, en representación de la civilización occidental y cristiana deben utilizar todos los medios para combatir el enemigo» (Auto del Juzgado de Instrucción número cinco de la Audiencia Nacional española del 11 de mayo de 1998, http://www.nuncamas.org/juicios/espania /espania_110598.htm). 
Pero las Madres no encontraron apoyo en lo público argentino. El «silencio social» que mantuvo oculto el asunto de los desaparecidos, dejó como saldo un público miedoso, que guardó silencio o difamó a las Madres como madres de los terroristas que ensuciaban la reputación de Argentina en el exterior y las cuales eran - a causa de sus propias protestas - víctimas de una persecución y de la pregunta con que se las insultaba: «¿Ustedes no son argentinas?» (CALVEIRO, 1998: 150; GuZMÁN BOUVARD, 1994: 156).

Las Madres no fueron el único grupo nacional que se dedicó al problema de los desaparecidos. También se formaron grupos tales como los «familiares» y las «abuelas» más algunas organizaciones religiosas, humanitarias y políticas; en especial debemos mencionar algunas organizaciones nacionales eclesiásticas, y otras formadas en cooperación con organizaciones internacionales, por ejemplo FEDEFAM, CLAMOR en Sao Paulo y el vicariato de solidaridad en Chile, así como también la «Liga» ${ }^{2}, \mathrm{APDH}^{3}$, $\mathrm{MEDH}, \mathrm{SERPAJ}{ }^{4}$, CELS ${ }^{5}$ y MJDH ${ }^{6}$. Pero en las organizaciones creadas por los damnificados - especialmente las Madres - la protesta alcanzó su expresión mas auténtica. La resistencia incansable de madres y amas de casa, y su presencia regular en la Plaza de Mayo fueron algo inesperado para los militares. No habían tenido en cuenta ese tipo de reacción (SCHOLZ, 1990: 128).

Así, las Fuerzas Armadas, a tan sólo ocho meses del inicio de la protesta de las Madres en la Plaza de Mayo, hicieron el primer intento por destruir esa expresión de protesta con las mismas medidas que habían ya usado efectivamente en la lucha contra la subversión: la desaparición de 12 mujeres — entre ellas Azucena Villaflor-y de dos monjas francesas, fue planificada y llevada a cabo por el Teniente de Marina Alfredo Astiz, después de que las Madres, algunas semanas antes, publicaran una solicitud en un diario con una lista de nombres de personas desaparecidas, ganando por primera vez la atención pública nacional acerca de un problema que hasta ese preciso momento no había sido abordado por ningún medio masivo de comunicación.

\subsection{Lo Público Transnacional}

Pero los militares no consiguieron escapar del asunto en silencio. Al contrario: el movimiento de protesta -internamente aún muy frágil y contando solamente con pocos militantes- se internacionalizó. Primero, las Madres ganaron la atención de la prensa internacional por estar presentes en cada visita de políticos extranjeros. Así las visitas de Terence Todman y Cyrus Vance fueron las primeras oportunidades en las que representantes de la prensa internacional se preguntaron quiénes eran esas mujeres (BONAFINI, 1988). Y aún cuando la Junta durante el Mundial de Fútbol de 1978 consiguió evitar una presencia mediática de las Madres mediante una supresión masiva, nuevos secuestros e intimidaciones ${ }^{7}$, el año del Mundial fue el año de la «mundialización» decisiva de la protesta.

${ }^{2}$ Liga Argentina por los Derechos Humanos (ver: VeIGA, 1985: 23).

3 Asamblea Permanente por los Derechos Humanos (VeIGA, 1985: 111).

${ }^{4}$ Servicio Paz y Justicia en América Latina.

${ }^{5}$ Centro de Estudios Legales y Sociales.

${ }^{6}$ Movimiento Judío por los Derechos Humanos.

${ }^{7}$ «Y viene la época del Mundial, en 1978. Ese horror que para nosotras era el Mundial y que a mucha gente los ponía contentos. Se provocaban más secuestros. Se acentuaba la represión. Se acentuaba en la Plaza. Nos 
El primer grupo de soporte en el exterior fue fundado por Liesbeth Den Uyl, la esposa del Primer Ministro de los Países Bajos (Hauck/HuHLe, 1996: 111). También el Grupo SOLMA ${ }^{8}$ en Francia comenzó con manifestaciones frente a la Embajada de Argentina en París. Las Madres viajaron a los Estados Unidos y a Europa solicitando colaboración a numerosas organizaciones no gubernamentales —entre ellas: Amnistía Internacional (AI, 1979; 1982; 1987), America’s Watch/Human Rights Watch (AW, 1991), Comisión Internacional de Juristas (International Commission of Jurists, 1975), Consejo de la Iglesia Mundial, Sociedad Internacional para los Derechos Humanos, PAX ROMANA, Pax Christ Internacional, Asociación Internacional contra la Tortura, Asociación de Juristas Democráticos (Association Internationale des Juristes Démocrates, 1976), Liga Internacional para la Defensa de los Derechos y la Liberación de los Pueblos, Penal Law Association y el grupo de Derechos de la Minoridad.

Hebe de Bonafini visitó en 1979 al Presidente italiano Sandro Pertini y al Papa. Continuaron los viajes a Suecia ${ }^{9}$, Canadá y Australia y el poder de la atención pública internacional empezó a proteger a las Madres de las amenazas de muerte (HAUCK/HuHLE, 1996: 111; SICILIANO, 1992: 21).

También en Alemania hubo protestas. El 26 de marzo de 1976, pocos días después del golpe de Estado en Argentina, «desapareció» Klaus Zieschank, estudiante de la Universidad Técnica de Munich ${ }^{10}$. Fue entonces que se formó la «Iniciativa para la Libertad de Klaus Zieschank» —una asociación fundada por miembros de Amnistía Internacional y estudiantes de la Universidad Técnica de Munich, que pronto fue apoyada por abogados, funcionarios de la Iglesia y algunos políticos-y que organizó una «huelga de hambre» en Bonn (CUYA, 2000). Similar repercusión logró el caso de Elisabeth Käsemann (WERZ, 1996: 303) y la campaña «Fútbol y Tortura», que promovió un boicot al Mundial de Fútbol en Argentina (Al caso Zieschank: THUN, 1985: 89).

Es digno de destacar que en el año 1980 se le concedió el Premio Nobel de la Paz a Adolfo Pérez Esquivel, fundador de la organización SERPAJ («Servicio paz y justicia» $)^{11}$. Esto es de especial relevancia en el contexto del proceso de globalización de la protesta, de una acción global de damnificados, actuantes espontáneos y organizados, gubernamentales y no gubernamentales puesta en lo público por los medios de comunicación. Así, en la ceremonia de entrega de premios, el comité del Premio Nobel noruego citó a Jorge Luis Borges, quien exigió que uno no debe guardar silencio sobre las personas que desaparecieron y quien constató, que «la represión misma es una forma de terrorismo» (The Norwegian Nobel Committee, 1989).

llevaban presas a cada rato. Nos golpeaban. Ponían perros en la Plaza. Nosotras llevábamos un diario enroscado para cuando nos echaban los perros. Nos tiraban gases. Habíamos aprendido a llevar bicarbonato y una botellita de agua. Para poder resistir en la Plaza. Todo esto lo aprendimos ahí, en esa Plaza. Mujeres grandes, que nunca habíamos salido de la cocina, habíamos aprendido lo que habían hecho tantos jóvenes antes. Luchar por ese pedacito de Plaza, luchar por ese pedacito de cielo que significaba nada más y nada menos que esto que tenemos hoy. Y el Mundial también fue muy terrible para nosotras. Fue muy terrible porque en el Mundial se tapó, o se quiso tapar, todo lo que estaba pasando» (BONAFINI, 1988).

8 «Solidarité avec les Méres de la Place de Mai d'Argentine», ver http://web.tiscali.it/WIN/051b.html.

${ }^{9}$ En referencia a la protesta sueca: GONE, 1996.

${ }^{10}$ En total hubo alrededor de 500 víctimas no-argentinas o con doble nacionalidad.

${ }^{11}$ Las Madres acompañaron a Esquivel (HAUCK/HuHLE, 1996: 116). 


\section{IUS}

¿Cómo hay que evaluar esa acción de la conciencia mundial? ¿Se genera el «derecho mundial sin Estado» (TEUBNER, 1996: 257; 1997) por producción no política? ¿Es ética solamente un título errado para esos procesos de escándalo de la sociedad, porque se trata «no solamente de problemas de conciencia o de un reconocimiento moral, sino también de una forma de Derecho, por la cual se buscan posibilidades de sanción» (LUHMANN, 1999: 250), de una forma post-moderna y neo-espontánea de «derecho vivo» a modo de Eugen EHRLICH, para quien el motor de la evolución del derecho está situado «no en el poder legislativo, no en el poder judicial, pero en la sociedad misma» (EHRLICH, 1913: 390)? —Ésa es la tesis esgrimida por Niklas LuHMANN: que el mecanismo central para la instauración del Derecho mundial es exactamente ése, el de la colére publique, concepción de Emile DuRKHEIM (DuRKHEIM, 1930: 118; LUHMANN, 1993: 30)—. Según las palabras de LuHMANN: «Por supuesto que también es una paradoja decir que los derechos son validados por su violación y el escándalo correspondiente (la colère publique de Durkheim). Pero tal vez es esa exactamente la paradoja actual en las turbulentas relaciones mundiales y en presencia de la pérdida de relevancia de los órdenes clásicos-estatales» (LuHMANN, 1995: 581; 1995: 243).

Hoy, la instauración del Derecho mundial se lleva a cabo en primera línea por ese mecanismo, y para LUHMANN ésta es la prueba de que «el orden del Derecho mundial se parece más a las formas de orden de las sociedades tribales, lo cual significa que el Derecho mundial debe prescindir de fuerzas de sanción organizadas y de la definición auténtica de violaciones del Derecho según reglas conocidas» (LuHmanN, 1995: 234; 1993; 1998: 99; 1999: 250; TEUBNER, 1996: 255).

\section{ORITUR}

Es necesario corregir la descripción de LUHMANN en el sentido de que a pesar de la soberanía de los estados, en el Derecho internacional público han evolucionado reglas generales en el área de los derechos humanos fundamentales (FISCHER-LESCANO, 2000a: 27). De esa manera, los global remedies — los así llamados «tribunales descentrales y centrales» ${ }^{12}$ - sentenciaron, o se encuentran aún investigando casos como los de Milosevic, Pinochet o los ex-militares de la Argentina, Carlos Guillermo Suárez Mason, Santiago Omar Riveros, Alfredo Astíz, Juan Bautista Sasiain y Pedro Alberto Durán Sáenz. La forma de descripción de LuHMANN es, sin embargo, importante para la reformulación jurídica de los mecanismos de instauración del Derecho mundial y para una democratización fundamental de ese derecho hoy aún hegemónico (BRUNKHORST,

${ }^{12}$ Estos tribunales tienen una jurisdicción que en épocas de soberanía absoluta estaba solamente asignada a los tribunales locales nacionales (local remedies). Para una explicación de los global remedies rules: FIsCHER-LESCANO, 2002b: 217; compárese también: George SCELLES, quien describió que los tribunales nacionales operan en una forma de dédoublement fonctionnel, para poder acentuar en ciertos casos el carácter internacional de esos tribunales: «Il en est de même des agents. Le juge national, lorsqu'il rend un jugement dans une affaire entre nationaux et étrangers ou entre étrangers, cesse d'être un juge national pour devenir un juge international. C'est une constatation à laquelle on ne prend pas toujours garde, mais dont l'observation démontre la réalité, car ce qui caractérise une opération juridique, ce n'est pas le caractère de l'agent qui l'accomplit mais sa nature intrinsèque» (SCELLE, 1932: 56; compárese: CASSESE, 1990: 210). 
2002), porque el proceso de escandalización en el paradigma argentino — vinculado en gran parte a las Madres de Plaza de Mayo y a las llamadas organizaciones no gubernamentales en Argentina, los Estados Unidos y Europa que las apoyan- es algo más que solamente una forma de presión política.

\subsection{Convenciones}

Así, por un lado, se le debe atribuir a la protesta en gran medida que la práctica de desaparición de personas no se encuentre prohibida solamente por ser un delito general de «crimen de lesa humanidad», sino que se haya especificado como una «prohibición de la práctica de desaparición» en el Derecho penal internacional: El movimiento de la protesta se dirigió continuamente a las instituciones del sistema político. «Miles de casos han sido presentados ante la ONU y la Organización de los Estados Americanos por grupos como la Asociación Federal de Latinoamérica de parientes de desaparecidos (FEDFAM) y otros» (MCINTYRE/GASTON, 2001: 3). Todos ellos condenaron la práctica de la desaparición forzada de personas ${ }^{13}$. La Asamblea General de la Organización de los Estados Americanos afirmó en 1983 que la práctica de la desaparición forzada de personas en las Américas [...] constituye un crimen contra la humanidad ${ }^{14}$. La misma evaluación se encuentra en una resolución de la Asamblea Interparlamentaria del Consejo de Europa (Europarat) y en varias declaraciones y resoluciones de la Asamblea General de la ONU o de organizaciones dependientes de la ONU, tales como del ECOSOC ${ }^{15}$. En 1996, la Comisión de Derecho Internacional Público ha introducido la práctica de la desaparición forzada como crimen en el Draft Code of Crimes against Peace and Security of Mankind y adjuntó como explicación: «La desaparición forzada no fue incluida como crimen contra la humanidad en instrumentos previos. Aunque este tipo de conducta criminal es un fenómeno relativamente reciente, el presente código propone su inclusión como crimen contra la humanidad dada su extrema crueldad y gravedad» (UN, 1996: nota al pie 135). También en el Estatuto de Roma, el estatuto de la Corte Penal Permanente ${ }^{16}$, la técnica de la desaparición es un crimen de lesa humanidad [art. 7, párrafo I (i) ]. Además vale nombrar las declaraciones de la Comisión de Derechos Humanos de la ONU y del Working Group on Enforced or Involuntary Disappearances ${ }^{17}$ creado por la Comisión de Derechos Humanos. Asimismo, la propuesta de la Convención Internacional sobre la Protección de todas las Personas ante la Desaparición Forzada (International Convention on the Protection of all Persons from Enforced

${ }_{13}$ Ver el reporte de la Comisión Interamericana de Derechos Humanos, 14 de diciembre de 1979, http://www.nuncamas.org/document/internac/cidh79/index.htm. Ver también, OEA/Ser.L/V/II.49, doc. 19 y la declaración de la OAS a la desaparición: AG/Res.443 (IX-0/79) del 31/10/1979; AG/Res.510 (X-0/80) del 27/11/1980; AG/Res.618 (XII-0/82) del 20/11/1982; AG/Res.666 (XIII-0/83) del 18/11/1983; AG/Res.742 (XIV-0/84) del 17/11/1984; AG/Res.890 (XVII-0/87) del 14/11/1987.

${ }^{14}$ AG/RES. 666 (XIII-0/83), del 18 de noviembre de 1983.

${ }^{15}$ Declaration on the Protection of all Persons from Enforced Disappearances, GV-Res. 47/133 of 18 de diciembre de 1992 (GA/47/49, S. 207ff.); ver también: Resolution 33/173, del 20 de diciembre de 1978 y la resolución de la ECOSOC, del 10 de mayo de 1979 (Res. 1979/38) y de la Subcommission for the Prevention of Discrimination and Protection of Minorities [Resolution 5B (XXXII), del 5 de septiembre de 1979].

${ }^{16}$ UN Doc. A/CONF. 193/9 (1998).

17 ECOSOC-Resolution 20 (XXXVI), del 29 de febrero de 1980. 
Disappearance) dice en su preámbulo: «Considerando que la desaparición forzada de personas ataca los más profundos valores de cualquier sociedad comprometida con el respeto por el imperio de la ley, los derechos humanos y las libertades fundamentales, y que la práctica sistemática o extensa de tales actos constituye un crimen contra la humanidad» ${ }^{18}$.

\subsection{Procesos Mundiales}

Para nuestra observación jurídica el hecho de que la protesta se dedicara a interponer denuncias por los hechos ante fiscalías en los Estados Unidos y Europa es más importante que la ya descrita presión política. Sobre todo la «Coalición contra la Impunidad $\gg^{19}$ interpuso numerosas denuncias, por las que los ex-militares argentinos se ven hoy confrontados con procesos ante los global remedies. (FISCHER-LESCANO, 2005; PARENTI, 2002; MCINTYRE/GASTON, 2001). Hubo varias causas penales sentenciadas en Europa ${ }^{20}$ y sobre todo cabe destacar dos juicios civiles: El proceso ante una Corte Regional norteamericana en California, en la causa Forti vs. Suárez Mason ${ }^{21} \mathrm{y}$-en un caso de desaparición en Honduras- la sentencia del 29 de julio 1988 de la Corte Interamericana de Derechos Humanos en el caso Velázquez Rodríguez ${ }^{22}$. En la decisión de este último caso la Corte condenó la desaparición de Ángel Manfredo Velázquez Rodríguez y obligó al Estado de Honduras a pagar una indemnización a los familiares de la víctima. La decisión de la Corte Regional norteamericana en el caso Forti vs. Suárez Mason fue basada en el Alien Tort Statute. En relación al derecho global de ser protegido contra la desaparición forzada, la Corte declara que en la comunidad internacional hay un consenso sobre la definición de la desaparición, que tiene dos elementos centrales: $a$ ) secuestro por el estado y $b$ ) rechazo de ese hecho por el Estado. «Desaparición», concluye la Corte, «es una violación de los derechos humanos, que son reconocidos universalmente» ${ }^{23}$.

\subsection{Lex Humana}

Por un lado, el proceso de escandalización representó una presión política-jurídica y fue en sí conditio sine qua non para la condena de crímenes y criminales. Pero por otro lado, el proceso de escandalización también es, en un ámbito más directo, la manifestación de una nueva forma de expectativa normativa de expectativas normativas a nivel mundial: la protesta ha hecho valer una nueva norma de Derecho mundial al formular que «desaparición es injuria mundial». Este es un ejemplo de «derecho vivo»

${ }^{18}$ Draft International Convention on the Protection of all Persons from Enforced Disappearance, http://www.disappearances.org/undocs/undoc18.html.

${ }^{19} \mathrm{http}: / /$ menschenrechte.org/coalicion.htm.

${ }^{20}$ La jurisdicción de esos global remedies se basa regularmente en los principios de jurisdicción personal (pasiva o activa) y jurisdicción universal (OEHLER, 1983: 155; BROWNLIE, 1998: 303). Con referencia a los tribunales internacionales: BUERGENTHAL, 2001: 267; SHELTON, 2000: 147.

${ }^{21}$ 1988, 694 F. Supp. 707, en STEINER/Alston, 2000: 1062.

22 I.L.M. 28 (1989), 294 y ss.

${ }^{23}$ Fragmentos de la decisión pueden encontrarse en Steiner/Alston, 2000: 1062. 
en la Bucovina global (TeUBNER, 1996: 255), y esa lex bumana ${ }^{24}$ del ius gentium postmoderno se ve expresada en primera línea por actores de la sociedad civil en cooperación con los medios de comunicación y podría ser reformulada en el Derecho internacional público como «derecho consuetudinario de la sociedad mundial» ( ¡y no: derecho internacional consuetudinario!). LUHMANN describe esa pluralidad genérica de normas diciendo que «Por otro lado, los "derechos humanos" se manifiestan como una especie de programa de recuperación de moda como nunca antes [...] Pero tal forma de expectación normativa de expectaciones normativas se encuentra en gran parte muy por fuera del mundo de las formas jurídicas establecidas y apunta también contra el Derecho. Legal o ilegal —humanidad es lo que vale-. Si se pudieran verificar estas interpretaciones y suposiciones en investigaciones empíricas, se demostraría que el Derecho practicado en el sistema jurídico difiere nuevamente del "derecho vivo"» (LUHMANN, 1995: 556).

Que el Derecho practicado en el sistema del Derecho internacional público regularmente aún ignore la lex humana no significa que los procesos de escándalo no sean también aquí, de todos modos, fenómenos jurídicos; es decisivo que se trata de operaciones que son capaces de transportar el símbolo de validez jurídica, el uso del código comunicativo legal/ilegal, asociado a un consenso atribuible a la sociedad civil a favor de un lado u otro de esta distinción (FISCHER-LesCANo, 2003; 2005). Para el Derecho internacional es importante reformular esa lex bumana, porque en caso contrario, decide obviando el medio ambiente social y pierde toda su atención por los individuos ${ }^{25}$, o corre también el peligro de ser víctima de la propia experiencia empírica de que hay que contar inevitablemente con revoluciones u otros cambios parciales de las estructuras de Derecho y política como posibles accidentes de la vida constitucional (TomUsCHAT, 1972: 100).

La perspectiva de que el Derecho internacional aún centrado en el Estado pueda evolucionar como Derecho mundial de la sociedad civil mundial, adaptándose estructuralmente a los cambios de las condiciones básicas de la sociedad mundial, no es una utopía. En ese sentido, la Magistrado especial belga Van den Wyngaert hizo referencia a los mecanismos de instauración del Derecho mundial por la sociedad civil con su opinión disidente en el caso «orden de detención», causa en la cual debía juzgar la Corte Internacional de Justicia de La Haya en febrero del 2002. La reformulación de esos mecanismos puede ser demasiado estrecha si se la restringe solamente a organizaciones no gubernamentales, pero su exposición es bienvenida como un primer paso para una reformulación de adecuada complejidad de los mecanismos de incorporación del Derecho de la sociedad mundial en el Derecho internacional. En las palabras de la Magistrado: «Organizaciones jurídicas como Amnistía Internacional, Abogados sin fronteras, Human Rights Watch, Federación Internacional de Ligas de Derechos Humanos (FIDH)

${ }^{24}$ En este caso, lex humana hace referencia (relacionada a concepciones de la lex mercatoria y la lex informática y en contraposición con la trilogía escolástica de lex aeterna - lex naturalis_lex bumana) a la instauración no estatal del derecho positivo, es decir, a los mecanismos de generación de normas de la sociedad mundial en contraposición con los mecanismos de generación de normas por el sistema político de la sociedad, en otras palabras, los de la «comunidad internacional».

${ }_{25}$ «En ese caso, el sistema de derecho necesitaría de escándalos efectivos en la prensa y de una "Amnistía Internacional" gigante para poder salvar al Estado del Derecho, por el cual ningún individuo siente ya interés propio» (LUHMANN, 1995: 489). 
y la Comisión Internacional de Profesionales del Derecho han tomado posiciones claras en el tema de la responsabilidad internacional. Esto debe ser visto como la opinión de la sociedad civil, una opinión que hoy no puede ser completamente descontada en la formación del Derecho internacional consuetudinario [...] La Corte comete un error al desconocer este desarrollo y no pone en discusión las fuentes relevantes» ${ }^{26}$.

\subsection{La Vigencia de la Ley en Argentina}

En la Argentina de hoy, cuando caminamos por nuestras calles, sabemos que nos cruzamos con asesinos y torturadores de la peor calaña, que se mueven entre nosotros con toda libertad (SÁBATO, 1996).

Si se entiende legitimidad como una disposición generalizada de aceptar decisiones indefinidas en contenido y de adaptarse en su aprendizaje (LuHMANN, 1969: 28), se reconoce el problema resultante de la exclusión e inclusión asimétrica que se les ha presentado a las élites en la Argentina posdictatorial bajo la impresión de la descrita colère publique mondiale y de las decisiones de los global remedies — sobre todo independientemente del proceso de transición histórica- («Vergangenbeitsbewältigung») al que se le da preferencia básica: en el clima social argentino se institucionalizó un no-reconocimiento de la impunidad de los militares ${ }^{27}$, desapareció la legitimación de las decisiones de impunidad ${ }^{28}$. Esa desaparición es un fenómeno que se da cuando «el concerniente busca sobrevivir en la protesta contra esa decisión, se resiste, revive siempre su derecho violado, se rasca la costra de sus heridas y busca organizar ayuda y aceptación contra esa decisión; en breve: no aprende sino que se queda en sus viejas y desilusiona-

${ }^{26}$ Opinión discrepante de la jueza van den Wyngaert, Corte Internacional de Justicia del 14 de febrero 2002, caso concerniente al «Arrest Warrant» del 11 de abril del 2000 (República Democrática del Congo vs. Bélgica), pp. 27 y ss.

27 En la época de la Junta Militar no había en principio, fuera del procedimiento babeas corpus generalmente sin éxito, ningún otro tipo de proceso jurídico que se ocupara de las víctimas de los desaparecidos y del grado de responsabilidad de aquellos que decidieron sobre su destino. Sin embargo, Argentina comenzó positivamente un proceso interno de transición organizado estatalmente durante los primeros años posteriores a la dictadura con la formación de la CONADEP, cuyo trabajo culminó con el informe «Nunca más». A pesar de que el 21 de septiembre de 1983, los militares aún hicieron el intento de otorgarse una auto amnistía, en la cual declararon caducos todos los delitos cometidos hasta el 17 de junio de 1982 en razón de la lucha contra la subversión, existieron también paralelamente a la CONADEP otros intentos procesales regulados nacionalmente contra los militares, dado que se declararon nulas las leyes de auto amnistía de la junta y el gobierno democrático de Alfonsín determinó por posibilitar la persecución penal contra los primeros nueve comandantes activos de Ejército, Aviación y Marina de la dictadura. En un gran proceso, en el que fueron interrogados 900 testigos y en el cual el actual jefe de la fiscalía de la Corte Criminal Internacional, Luis Moreno Ocampo, que en aquel entonces actuara como representante de la fiscalía argentina, se condenó de hecho a cinco de estos nueve comandantes por asesinato, privación de la libertad, tortura, forzamiento y robo a prisiones de largos años, en parte a cadena perpetua. Estos procesos, sin embargo, aún en los casos donde los tribunales argentinos se ocupaban de los desaparecidos, acababan hasta 1995 regularmente en la impunidad de los autores; el gobierno de Alfonsín (razones para esta decisión: ALFONSín, 1993: 15) determinó nuevas leyes de amnistía ya en los primeros tiempos de su período legislativo, luego de haber caído bajo fuerte presión por parte de los militares: la Ley de Punto Final (del 29 de diciembre de 1986, Legislación Argentina, año 1986-B, p. 1100) y la Ley de Obediencia Debida (publicado el 9 de junio de 1987, Boletín Oficial, Buenos Aires). Atribuciones de culpas individuales no se llevaban a cabo por lo regular. Si hubo condenas, como en el caso de los cinco comandantes, generalmente solían ser indultados (AMBOS, 1997: 75; SANCINETTI, 1988).

${ }_{28}$ Sobre los problemas de legitimación en la fase de transición en Argentina en general: HALDENWANG, 1996: 177. 
das expectativas» (LuHMANN, 1969:33). Mientras que esto se limite a casos específicos y singulares - como en la fase hasta 1995, en la cual las protestas no fueron apoyadas por la sociedad argentina-, todo aquél que quiera resistirse a esas disposiciones obligatorias no puede contar con el apoyo de otros: «La propia resistencia se le atribuye a él mismo y no al fracaso de las instituciones. Así se la entiende como obstinación, manía de criticar, falta de razonamiento o por lo menos como una posición fuera de lo común, prácticamente irracional. Bajo ese tipo de interpretación, el caso en cuestión no puede expandirse, sino que permanece adherido a él» (LuHmanN, 1969: 123). Las Madres Locas (BousQueT, 1983: 170).

Pero las protestas se expandieron. El demos argentino no se contentó con la modificación del código argentino de legalidad a través del de exclusión (VEIRAs, 2000). El apoyo simbólico insuficiente de las desilusionadas expectativas no llevó a los ciudadanos a aceptar los nuevos hechos reales como normas, sino a buscar nuevos foros de confirmación de sus expectativas en contra de esos hechos; foros que se encontraron a través de la inclusión en el sistema de los derechos humanos universales y de los global remedies. Así se creó primeramente un clima social global, luego local, que no estaba dispuesto a aceptar las decisiones con las cuales se quería acabar con las investigaciones de la desaparición de personas (Risse/Ropp, 1999: 234) Las leyes de amnistía del gobierno del presidente Alfonsín, su justificación por los jueces de la Corte Suprema de la Nación ${ }^{29}$ y su confirmación por los decretos de los presidentes Menem ${ }^{30}$ y De la Rúa ${ }^{31}$ fueron presionadas. Y con la liberación de Oliveira de prisión preventiva italiana en septiembre del 2000 y su fuga desde Italia al refugio de la impunidad, se intensificaron las demandas de anulación de las leyes de amnistía: «dichos procesos han tenido una especial repercusión en los países latinoamericanos. Que los crímenes cometidos en esta región comenzaran a ser investigados y discutidos a nivel mundial, que se dictaran condenas (in absentia) y órdenes de captura contra los responsables, que jueces de distintos países solicitaran de modo incesante la extradición de personas acusadas de crímenes gravísimos, todo ello, ha contribuido a mantener vigente la expectativa de que los hechos finalmente sean juzgados. La existencia de juicios en otros países contrasta con la política de impunidad que hasta el momento prevalece en nuestro país y provoca que Argentina sea vista como un lugar de refugio de personas acusadas por crímenes contra la humanidad. En este sentido, el ejercicio de la jurisdicción extraterritorial ha tenido un claro efecto estigmatizador de la política del Estado argentino en la materia» (PARENTI, 2002).

El cambio de clima social ya había llevado a una ola de nuevos procesos en Argentina desde 1995. La presión pública se afianzó adicionalmente a través de las expresiones de algunos militares referentes a los hechos durante la dictadura. El presidente Menem intentaba proteger a los militares y se adhirió a las afirmaciones de que el régimen

${ }^{29}$ La Corte decidió el 22 de junio de 1987: «En consecuencia de la deliberación que antecede el Tribunal resuelve: 1) se rechazan los planteos de inconstitucionalidad de la ley 23.521 efectuados por los representantes de los particulares damnificados; 2) se declara que la ley 23.521 comprende a los imputados Etchecolatz, Bergés y Cozzani, a quienes se absuelve disponiendo su inmediata libertad, a cuyo fin se oficiará al señor Ministro de Defensa» (http://www.desaparecidos.org/arg/doc/secretos/corte.html).

${ }^{30}$ Decretos n. $^{\text {os }} 1002,1003,1004,1005$ del 7.10.1989 y 2741, 2742, 2743, 2744, 2745, 2746, del 30 de diciembre de 1990.

31 Decreto n. ${ }^{\circ} 1581$, de diciembre del 2001. 
militar sólo se había querido defender de la vigencia de la ley (VERBITSKY, 1995: 14). Pero luego de las expresiones del antiguo capitán de marina Adolfo Scilingo en una entrevista con el periodista Horacio VERBITSKY ${ }^{32}$, publicado en su libro El Vuelo, las dudas expuestas públicamente sobre la legalidad de la política de impunidad no pararon más (AMBOS, 1996: 77; CELS, 1995: 128). En esa entrevista, Scilingo prestó declaraciones acerca de cómo se adormeció a entre 1.500 y 2.000 presos en los campos de concentración de la ESMA y se los arrojó vivos desde aviones en el océano atlántico. (VERBITSKY, 1995: 17).

La ONG argentina Centro de Estudios Legales y Sociales (CELS) se decidió entonces a interponer nuevas demandas que exigieran un derecho por la verdad y con las cuales se quería aclarar al destino de los desaparecidos. Pero aún esos juicios por la verdad irían a fracasar en su comienzo frente a los tribunales argentinos. De esta manera los rechazos de los tribunales inferiores se extendieron a través de los años y de diferentes instancias, y el 13 de agosto de 1998, la Corte Suprema de Justicia de la Nación acabó por determinar, a lo largo de catorce renglones para los cuales necesitó tres años, que no existía una base jurídica para estos juicios por la verdad, pues las leyes de amnistía igualmente estaban en contra de una persecución de los autores de los crímenes (BRETT, 2001: 4). Pero los damnificados tampoco se contentaron con esto, sino que buscaron el camino hacia los global remedies. En noviembre de 1998, Carmen Aguiar Lapacó interpuso una demanda en la Comisión Interamericana de Derechos Humanos, la cual fue aprobada por esta comisión en mayo de 1999, y la que llegó el 15 de noviembre de 1999 a su liquidación por igualación de proceso, en la cual Argentina se obligaba a aceptar y garantizar el derecho por la verdad ${ }^{33}$. Innumerables procesos como éste fueron (y aún se encuentran) perpetuados en épocas posteriores en Argentina (KoOY/CuRIA, 2000): en La Plata se realizaron entrevistas semanales de testigos, en las cuales se consiguió aclarar el destino de más de 2000 desaparecidos. Otros procesos en Buenos Aires, Bahía Blanca y Córdoba también llamaron la atención ${ }^{34}$. Y las organizaciones no gubernamentales cuentan con otro éxito: la instalación de la Comisión Nacional de Identidad (CONADI), que fue conformada por las Abuelas y sus abogados, fiscales argentinos y el encargado de los derechos humanos del gobierno de Menem. De los 240 casos de hijos desaparecidos, las Abuelas pudieron resolver los paraderos de 71 niños con el apoyo de expertos forenses y del Banco Nacional de Genes. Éstos habían nacido en parte en los campos de concentración de Campo de Mayo, El Pozo Banfield y Automotores Orletti y pasaron a formar parte de familias que, en algunos casos, los adoptaron de buena fe y, en otros, habían sido adoptados por criminales militares. Y gracias a que los actos criminales con respecto a los secuestros de hijos de desapareci-

32 Scilingo repitió estas expresiones el 7 de octubre de 1997 como testigo frente al juez español Garzón. A causa de su participación en los «vuelos de la muerte» Garzón le ordenó prisión preventiva. Así se encontró detenido en primera medida en Carabanchel, Madrid. Entre enero de 1998 y el 31 de julio del 2001 quedó libre bajo caución, entretanto Garzón determinó nuevamente prisión preventiva (BRETT, 2001: 8). Un sumario sobre las decisiones en el caso Scilingo y los otros procesos en España se encuentra bajo: http://www.nuncamas.org/juicios/juicios.htm; instructivamente: FEITLOWITZ, 1998: 193.

${ }^{33}$ Comp. BRETT, 2001: 4. Esto era de prever, pues en un fallo anterior [Manuel Stalin Bolaños $v$. Ecuador, Caso 10.580, Informe n. ${ }^{\circ}$ 10/95, Inter-Am.C.H.R., OEA/Ser.L/V/II.91 Doc. 7 at 79 (1996)] la comisión determinó las obligaciones de estados con respecto a ese tipo de hechos en relación a la decisión de la Corte Interamericana de Derechos Humanos en el caso Velásquez Rodríguez del 29 de julio de 1988.

34 Condenas por estos crímenes no hubo (GUTIÉRREZ, 2001; BRETT, 2001: 4). 
dos no se encontraban incluidos en las leyes de amnistía, se pudo llegar a condenas y detenciones ${ }^{35}$.

\subsection{Manifiesto Autopoiético}

Ese monismo jurídico comunicativo en el pluralismo jurídico genérico de la sociedad mundial (acerca de ese concepto: FisCHER-LESCANO/TEubNER, 2004; TEUbnER, 2003: 1; FISCHER-LESCANO, 2003; GÜNTHER/RANDERIA, 2001: 96) tuvo otra expresión aún más significativa en el sistema jurídico local argentino ${ }^{36}$ : el 6 de marzo de 2001 el juez federal Gabriel Cavallo declaró nulas las leyes de amnistía argentinas: «Por otra parte, como se verá, la República Argentina se ha integrado, desde sus albores, a la comunidad internacional, ha contribuido a la formación del Derecho penal internacional y ha reconocido la existencia de un orden supranacional que contiene normas imperativas para el conjunto de las naciones (ius cogens). [...] Por todo lo expuesto, resuelvo: I. Declarar inválido $[\ldots ..] \gg^{37}$.

Se trata de una decisión confirmada tanto por la Corte Suprema ${ }^{38}$ como por varios procesos paralelos ${ }^{39}$, los que a su vez han llevado a numerosos nuevos procesos en la República Argentina. En la literatura jurídica (PARENTI, 2003) esto es conocido como el manifiesto autopoiético (TEUBNER, 1996: 255; 1989; FISCHER-LESCANO, 2005) del Derecho ${ }^{40}$, el cual aún debe bregar por su propia autonomía frente a la política en un plébiscite de

35 Por lo menos temporalmente bajo prisión o prisión domiciliaria: Jorge Rafael Videla, junio de 1998; Emilio Eduardo Massera, noviembre de 1998; Jorge Antonio Acosta, diciembre de 1998; Antonio Vañek, diciembre de 1998; Héctor Antonio Febres, diciembre de 1998; Oscar Rubén Franco, diciembre de 1998; José Suppich diciembre de 1998; Reynaldo Benito Bignone, enero de 1999; Cristino Nicolaides, enero de 1999; Policarpo Vázquez, marzo de 1999; Carlos Guillermo Suárez Mason, diciembre de 1999; Juan Bautista Sasiaiñ, marzo de 2000; Santiago Omar Riveros, agosto de 2000; Miguel Osvaldo Etchecolatz, abril del 2001; Jorge Antonio Bergés, abril del 2001 (AMNISTÍA INTERNACIONAL, 2001).

36 Sobre la colaboración informal de los jueces en el Derecho mundial: SLAUGHTER, 1997: 186; sobre el Derecho argentino con respecto a las leyes de amnistía: MATTAROLLO, 2001: 135.

37 Fallo dictado in re «Simón, Julio, Del Cerro, Juan Antonio s/sustracción de menores de 10 años», causa n. ${ }^{\circ} 8686 / 2000$ del Juzgado Nacional en lo Criminal y Correccional Federal n. ${ }^{\circ} 4$ de la Capital Federal, Secretaría n. ${ }^{\circ}$ 7. Publicada en Revista Argentina de Derecho Constitucional, Buenos Aires, Ediar, Año II, 2001, n. ${ }^{\circ} 3$, 129 y ss. (/252); vea también la confirmación por la Cámara Federal de Apelaciones de la Capital Federal, Fallo dictado por la Sala II, el 9 de noviembre de 2001 in re «Incidente de apelación de Simón, Julio» (reg. 19.193). La Cámara Federal afirmó la contradicción de las leyes 23.492 y 23.521 con los tratados internacionales (ver también: PARENTI, 2003).

${ }^{38}$ Corte Suprema, 14 de junio de 2005, una causa en la que intervienen el Centro de Estudios Legales y Sociales (CELS) y las Abuelas de Plaza de Mayo.

${ }^{39}$ Causa n. ${ }^{\circ}$ 18.062, Espinoza Bravo, Pedro Octavio s/procesamiento, Jdo. Fed. n. ${ }^{o}$ 1, Sec. n. ${ }^{\circ}$ 2., Registro n. ${ }^{\circ}$ 19.338; ver el sumario sobre los fallos en Argentina: http://www.nuncamas.org/juicios/juicios.htm.

${ }^{40}$ En este contexto también es importante agregar que desde julio del 2003 el sistema político argentino se ha reapropiado del problema de la amnistía. Como primera medida, el presidente argentino Néstor Kirchner derogó el decreto 1581 firmado en diciembre del 2001 por Fernando de la Rúa, que impedía la extradición de argentinos acusados de violaciones a los derechos humanos. La decisión se aceleró tras el pedido del juez español Baltasar Garzón de extraditar a 46 ex represores argentinos. Pocos días después de esta derogación, por lo menos 43 de ellos se encontraban ya a disposición de la justicia. Entre los militares e integrantes de las fuerzas de seguridad reclamados por el juez español, se hallan integrantes emblemáticos de la última dictadura militar como Jorge Rafael Videla, Emilio Massera, Antonio Domingo Bussi, Guillermo Suárez Mason o el ex capitán Alfredo Astiz (BLETA, 2003). En los siguientes días, el gobierno argentino ratificó la convención sobre la imprescriptibilidad de los crímenes de guerra y lesa humanidad (adoptada y abierta a la firma, ratifica- 
tous les jours: «La politización, por ejemplo, es interminable, incluso si nunca puede ni debe ser total. Para que esto no sea una perogrullada o una trivialidad, es necesario reconocer la siguiente consecuencia: cada avance de la politización obliga a reconsiderar, es decir, a reinterpretar los fundamentos mismos del derecho tal y como habían sido calculados o delimitados previamente. Esto fue cierto en la Declaración de los Derechos del Hombre, en la abolición de la esclavitud, en todas las luchas emancipatorias que están y deberán estar en curso, en todo el mundo, para los hombres y para las mujeres. Nada me parece menos periclitado que el ideal emancipatorio clásico» (DERRIDA, 1992: 28).

\section{BIBLIOGRAFÍA}

Alfonsín, 1993: «"Never Again" in Argentina», en Journal of Democracy, 4, pp. 15 y ss.

Aмвоs, 1996: «Ich fühle mich als Mörder», Die Bekenntnisse des (ehem.) Korvettenkapitäns Francisco Scilongo, en NOLTE (ed.), Vergangenheitsbewältigung in Lateinamerika, Francfort a. M., pp. 77 y ss.

- 1997: Straflosigkeit von Menschenrechtsverletzungen. Zur «impunidad» in südamerikanischen Ländern aus völkerstrafrechtlicher Sicht, Freiburg.

AI, 1979: The «disappeared» of Argentina, London.

- 1982: The State of Siege and Political Imprisonment in Argentina, London.

- 1987: Argentina: The military juntas and buman rights, London.

- 2001: Report: AI-index: IOR 53/004/2001, London.

Association InTERnATIONALE Des JuRistes DÉmocrates, 1976: La Repression en Argentine, Brüssel.

AW, 1991: Truth and partial justice in Argentina, an update, New York.

BLETA, 2003: Kirchner derogó el decreto que impedía extradiciones, El Clarin, 26 de julio de 2003.

BONAFINI, 1988: «Historia de las Madres de Plaza de Mayo. Conferencia pronunciada el 6 de julio de 1988 por Hebe de Bonafini, presidenta de la Asociación Madres de Plaza de Mayo», http://www.Madres.org/aleman/historia/contenido/historia.htm.

BousQueT, 1983: Las locas de la plaza de Mayo, Buenos Aires.

BRETT, 2001: en HRW, «Argentina: Reluctant Partner», Reports, 2001, vol. 13, Issue 5 (B).

BROwnLIE, 1998: Principles of Public International Law, 5.a ed., Oxford.

BRUNKHORST, 2002: Solidarität. Von der Bürgerfreundschaft zur globalen Rechtsgenossenschaft,

Francfort a. M.

BrusChteIn, 1997: El día que ellas empezaron, en p. 12, del 29 de abril de 1997.

BUERGENTHAL, 2001: «Proliferation of International Courts and Tribunals: Is It Good or Bad?», in: Leiden Journal of International Law, 14, pp. 267 y ss.

ción y adhesión por la Asamblea General en su resolución 2391 [XXIII], del 26 de noviembre de 1968), que ya había sido aprobada por Argentina a través de la Ley 24.584 en 1995 en el Congreso Nacional, lo cual llegó a su punto máximo con la decisión de la Cámara de Diputados convocada por los presidentes de las diferentes fracciones parlamentarias, que declaró «insanablemente nulas» ambas leyes de amnistía (EICHELBAUM, 2003). A causa de la preexistente nulidad de las leyes de amnistía — por haber significado una violación de las obligaciones internacionales de la República Argentina (MatTarollo, 2001: 96; PAREnTI, 2003; KoKOTT, 1987: 506) -, es que las recientes decisiones, como la de la Cámara de Diputados, eran, desde el punto de vista político, de gran importancia, pero solamente de valor declarativo. Consecuentemente, la Corte Suprema ha determinado confirmar definitivamente la nulidad de las leyes de amnistía, invocando una violación del Derecho internacional público. 
Calveiro, 1998: Poder y Desaparición, Buenos Aires.

CASSESE, 1990: «Remarks on Scelle's Theory of "Role Splitting" (dédoublement fonctionnel), in International Law», en EJIL, 1, pp. 210 y ss.

Centro de Estudios Legales y Sociales, 1995: Informe Anual sobre la situación de los derechos bumanos en la Argentina, Buenos Aires.

CONADEP, 1984: Nunca Más. Informe de la Comisión Nacional sobre la Desaparición de Personas, Buenos Aires.

CuYA, 2000: Die Überwindung der Vergangenheit in Argentinien: Bemühungen gegen die Straflosigkeit nach 23 Jahren, http://www.menschenrechte.org/NMRZ/artikel3.htm, Nürnberg.

DERRIDA, 1992: «Force of Law. The "Mystical Foundation of Authority" », en DruCILla CoRNELl, M., y Rosenfeld, D. C. (eds.), Deconstruction and the Possibility of Justice, New York, pp. 3 y ss; versión espanola: Fuerza de Ley. El fundamento místico de la autoridad, traducción de Baberá, A., y PeÑalver Gómez, P., Madrid 1997.

DuRKHEIM, 1930: Über soziale Arbeitsteilung. Studie über die Organisation böherer Gesellschaften, 3. a ed., Francfort a. M., 1999.

EHrLICH, 1913: Grundlegung der Soziologie des Rechts, 3. . ed., Berlin, 1967.

Eichelbaum, 2003: «Diputados aprobó la nulidad de la Obediencia Debida y el Punto Final», El Clarín, 13 de agosto de 2003.

FeITlOWITZ, 1998: A Lexicon of Terror. Argentina and the Legacies of Torture, Oxford.

FISCHER-LeSCANO, 2002a: «Globalización de los derechos humanos», en Pasajes de Pensamiento Contemporáneo, 10, Valencia, pp. 27 y ss.

— 2002b: «Globalverfassung: Los desaparecidos und das Paradox der Menschenrechte», en Zeitschrift für Rechtssoziologie, 2/23, pp. 217 y ss.

— 2003: «Die Emergenz der Globalverfassung», en Zeitschrift für ausländisches öffentliches Recht und Völkerrecht, 3/63, pp. 717 y ss.

- 2005: Globalverfassung. Die Geltungsbegründung der Menschenrechte, Weilerswist.

FIsCHER-LeSCANO/TEUBNER, 2004: «Regime-Collisions: The Vain Search for Legal Unity in the Fragmentation of Global Law», en Michigan Journal of International Law, 25: 4, pp. 999 y ss.

Gone, 1996: Dagmar Killer unmasked?, First Page 14 de abril de 1996.

GÜNTHER/RANDERIA, 2001: «Recht, Kultur und Gesellschaft im Prozess der Globalisierung, Werner Reimers Stiftung, Schriftenreihe: Suchprozesse für innovative», Fragestellungen in der Wissenschaft, 4, Bad Homburg.

GutiéRrez, 2001: Menéndez se negó a declarar y la jueza lo dejó detenido, p. 12, 29 de abril de 2001.

GuZMAN BOUVARD, 1994: Revolutionizing Motherhood, Wilmington.

HaldeNWANG, 1996: «Der Anpassungsprozeß und das Problem der Legitimierung», en NolTE (ed.), Argentinien: Politik, Wirtschaft, Kultur und Außenbeziebungen, Frankfurt a. M., 177 y ss.

Hauck/Huhle, 1996: «20 Jahre Madres de Plaza de Mayo», en Nolte (ed.), Vergangenheitsbewältigung in Lateinamerika, Francfort a. M., pp. 108 y ss.

InTERNATIONAL COMmission OF JuRISTS, 1975: The Application in Latin America of International Declarations and Conventions relating to Asylum, Genf.

КокОтт, 1987: «Völkerrechtliche Beurteilung des argentinischen Gesetzes Nr. 23.521 über die Gehorsamspflicht (obediencia debida)», Zeitschrift für ausländisches öffentliches Recht und Völkerrecht, pp. 506 y ss.

KoOy/Curia, 2000: «Brinzoni: los juicios por la verdad no lograron nada», El Clarín, 26 de julio de 2000 . 


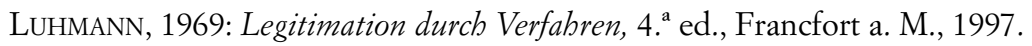

- 1993: «Gibt es in unserer Gesellschaft noch unverzichtbare Normen?», Heidelberger Universitätsreden, 4, Heidelberg.

— 1995: «Das Paradox der Menschenrechte und drei Formen seiner Entfaltung», in LuHMANN, Soziologische Aufklärung, 6, Die Soziologie und der Mensch, Opladen, pp. 229 y ss.

- 1995: Das Recht der Gesellschaft, Francfort a. M.

- 1998: «Meinungsfreiheit, öffentliche Meinung, Demokratie», en LAMPE, E.-J. (ed.), Meinungsfreibeit als Menschenrecht, Interdisziplinäre Studien zu Recht und Staat, 9, Baden-Baden, pp. 99 y ss.

— 1999: «Ethik in internationalen Beziehungen», en Soziale Welt, 50, pp. 250 y ss.

Mattarollo, 1985: «Noche y niebla», El Periodista, 3/5-9/5/1985, 10.

- 2001: «La jurisprudencia argentina reciente y los crímenes de lesa humanidad», en Revista Argentina de Derechos Humanos, Buenos Aires: Ad-hoc, pp. 93 y ss.

MCINTYRE/DE GASTON, 2001: Enforced Disappearance of Persons: Its Inclusion as a Crime against Humanity in the ICC, www.law2.byu.edu/lwb/papers/disappear1 INAL.PDF.

OEHLER, 1983: Internationales Strafrecht. Geltungsbereich des Strafrechts, Internationales Rechts-

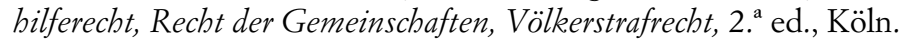

PARENTI, 2002: Nuevas perspectivas en el tratamiento penal de las violaciones de DDHH en Argentina entre 1976 y 1983, publ.: http://www.lainsignia.org/2002/mayo/der 012.htm.

RISSE/ROPP, 1999: «International human rights norms and domestic change: conclusions», in Risse/Ropp/SikKInK (Hrg.), International Norms and Domestic Change, Cambridge, pp. 234 y ss.

SÁBATO, 1996: cit. de GARCiA, Garzón, Argentina y la Communidad Internacional, El País, del 30 de noviembre de 1996.

SANCINETTI, 1988: Derechos Humanos en la Argentina post-dictatorial, Buenos Aires.

SCELle, 1932: Précis de droit des gens, vol. I, Principes et systématique, Paris.

SCHOLZ, 1990: Politische Kultur, soziale Bewegungen und Gewalt. Die gesellschaftlichen Bedingungen des Erfolges der Menschenrechtsbewegung in Argentinien, Berlin.

SHELTON, 2000: Remedies in International Human Rights Law, Oxford.

SicilianO, 1992: «Qui, gente di Plaza de Mayo», en Società civile, pp. 6, 21.

Slaughter, 1997: «The Real New World Order», Foreign Affairs, pp. 76, 183 y ss.

SONDERÉGUER, 1985: «Aparición con Vida. El movimiento de derechos humanos en Argentina», en JeLIN (ed.), Los nuevos movimientos sociales, Buenos Aires, pp. 1 y ss.

Steiner/Alston, 2000: Human Rights in Context. Law, Politics, Morals, 2. ${ }^{a}$ ed., Oxford.

TEuBNER, 1989: Recht als autopoietisches System, Frankfurt am Main, 2. ${ }^{a}$ ed., 1996.

- 1996: «Globale Bukowina: Zur Emergenz eines transnationalen Rechtspluralismus», en Rechtsbistorisches Journal, 15, 255 y ss.

- 1997: Global Law Without A State, Dartmouth.

— 2003: «Globale Zivilverfassungen: Alternativen zur staatszentrierten Verfassungstheorie», en Zeitschrift für ausländisches öffentliches Recht und Völkerrecht, pp. 1 y ss.

The Norwegian Nobel Committee, 1989: The Nobel Peace Prize 1980, http://www.nobel.se/peace/laureates/1980/press.html.

Thun, 1985: Menschenrechte und Außenpolitik. Bundesrepublik Deutschland-Argentinien 19761983, Bremen.

TOMusCHAT, 1972: Verfassungsgewohnheitsrecht? Eine Untersuchung zum Staatsrecht der Bundesrepublik Deutschland, Heidelberg. 
UN, 1996: International Law Commission Report, Draft Code of Crimes against the Peace and Security of Mankind, http://www.un.org/law/ilc/texts/dccomfra.htm.

VeIGA, 1985: Las organizaciones de derechos humanos, Buenos Aires.

VEIRAS, 2000: «Cinismo soberano», en p. 12 vom 20 de septiembre de 2000.

Verbitsky, 1995: El Vuelo, Buenos Aires.

WERZ, 1996: «Die deutsch-argentinischen Beziehungen», en NOLTE/WERZ (ed.), Argentinien. Politik, Wirtschaft, Kultur und Außenbeziebungen, Francfort a. M., pp. 297 y ss. 